\title{
3D Collapse and Accretion in Slowly Rotating Polytropes
}

\section{Aldo Batta and William H. Lee}

Instituto de Astronomía, UNAM - Ciudad Universitaria, México

E-mail: battadastro.unam.mo

Several tests have been made using the code GADGET-2, to model the collapse and accretion of a Polytropic envelope onto a $\mathrm{BH}$ of $1 M_{\odot}$. Based on previous results, a minimal resolution $\left(N_{S P H}=50000\right)$ is chosen and simulations are carried out with different initial angular momentum distributions. These will provide information about the formation of accretion disks at different distances from the BH. By observing the accretion of a non rotating, low internal energy $U_{\text {int }}$ polytropic envelope, we tested the Paczynski-Wiita potential used to account for some relativistic effects on the gas due to the $\mathrm{BH}$. These series of tests will help develop the necessary modifications to GADGET-2 in the context of the collapsar model for GRBs.

25th Texas Symposium on Relativistic Astrophysics - TEXAS 2010

December 06-10, 2010

Heidelberg, Germany 


\section{Introduction}

Though collapse and accretion of rotating material in astrophysical systems is a quite common issue, it hasn't been yet completely studied. This is due to the complexity of the systems involved, which sometimes include a complicated EOS for the collapsing and accreted material, as well as non trivial heating and cooling mechanisms that take place at high densities and temperatures. Due to these physical complexities the differential equations for hydrodynamics are difficult to solve and this translates in only few works done in 3D which include rotation effects, self-gravity and the instabilities that might come from them.

In order to make a "full" 3D study of the collapse and accretion for rotating material in an astrophysical problem, we consider the collapsar scenario [䧃] [园], which consists on a PreSN star whose $\mathrm{Fe}$ core and outer envelope, are about to collapse due to the shutdown of nuclear reactions in the central region and the $\mathrm{Fe}$ core reaching the Chandrasekhar mass. In our scenario, the $\mathrm{Fe}$ core collapses directly to form a $\mathrm{BH}$ which accretes the infalling outer envelope. To model gas dynamics, the simulations of this scenario will be made using the code GADGET-2 based on SPH (Smoothed Particle Hydrodynamics) [四]. In this work we show the current modifications to the code and some tests made to account for the accretion onto a $\mathrm{BH}$ and the pseudo-relativistic potential considered for the compact object.
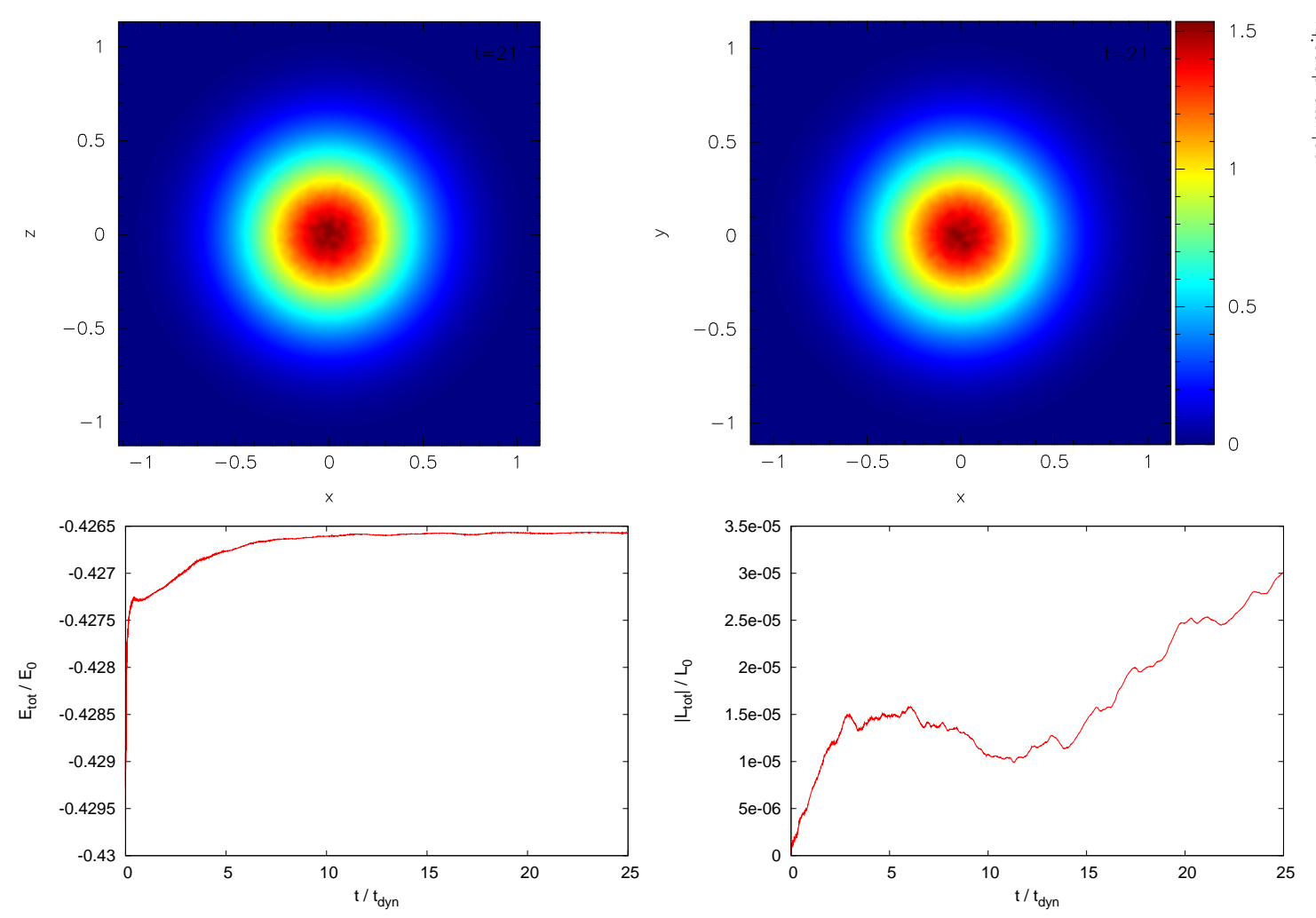

Figure 1: On the top panel the polytropic star column density after 21 dynamical time scales along the $Y$ (left) and the $Z$ (right) axis both without visible symmetry breaking. On the bottom the total energy (left) and angular momentum (right) with a very good conservation. 


\section{Stability tests}

Before starting with the collapsar model, we tested whether the code could properly solve the stationary solution of a non-rotating $\gamma=5 / 3$ polytrope, without having instabilities break the expected spherical symmetry. For $2 M_{\odot}$ polytropic stars with central density $\rho_{c} \simeq 2.5 \times 10^{9} \mathrm{~g} \mathrm{~cm}^{-3}$ we tested over several dynamical time scales (at least 10 ) whether there was any visible symmetry breaking or not. For these tests we used natural units by considering $G=1$ and scaling all units with the mass $M_{p}=2 M_{\odot}=3.98 \times 10^{33}$ [g], length $R_{p}=1.3099 \times 10^{8}[\mathrm{~cm}]$ and dynamical time scale $t_{d y n}=\left(R_{p}^{3} / G M_{p}\right)^{1 / 2}=0.092$ [s] of the system. Figure $\mathbb{U}$ shows the column density along the $Y$ (left) and $Z$ (right) axis of such a polytropic star with $\gamma=5 / 3$ after 21 dynamical time scales. The results show no visible symmetry breaking and there is a very good energy and total angular momentum conservation of $1 / 100$ and $1 / 100000$ respectively (bottom panel of Figure $\mathbb{W}$ ).
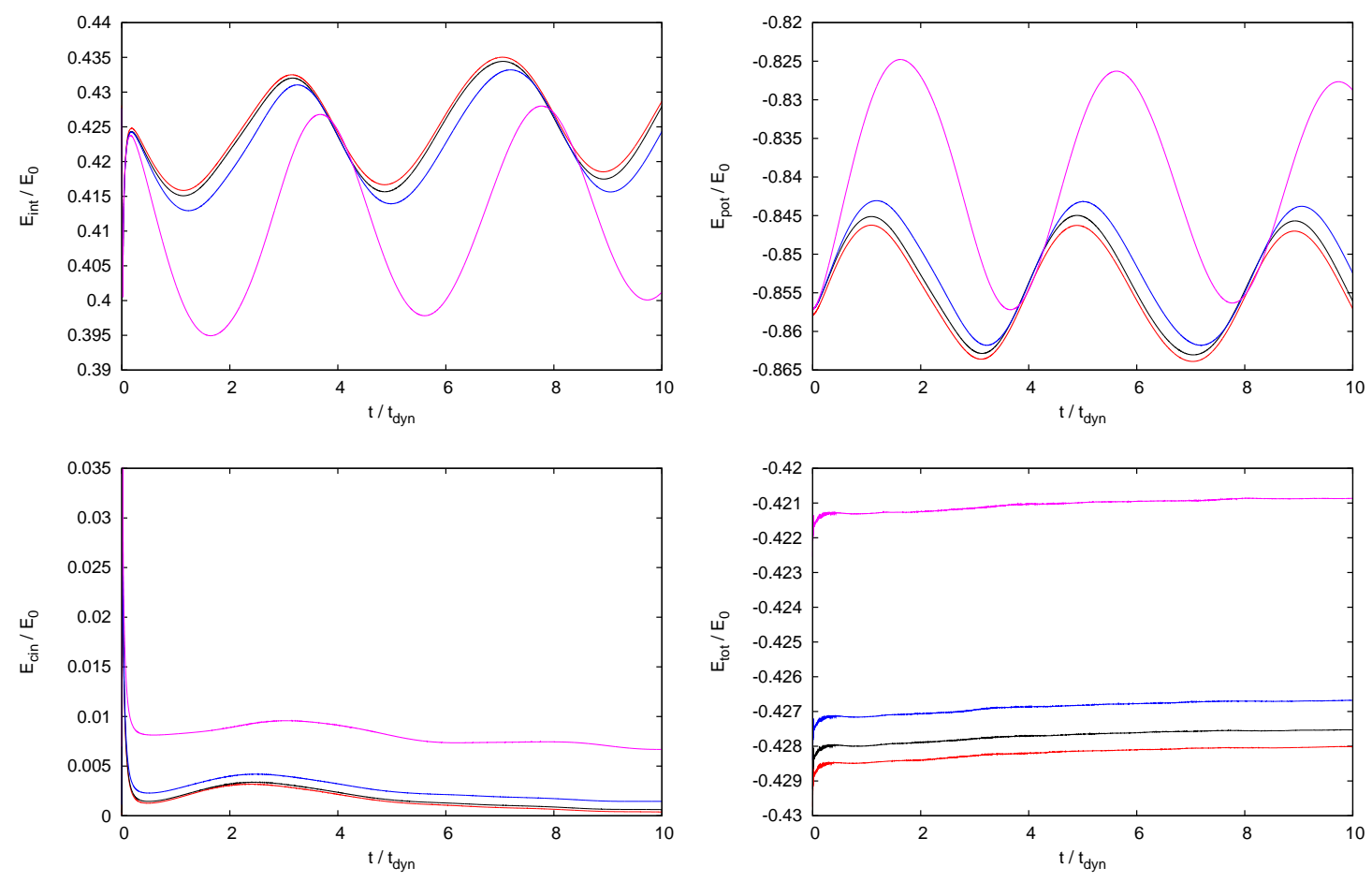

Figure 2: Normalized potential energy $E_{\text {pot }}$ (up-right), internal energy $E_{\text {int }}$ (up-left), kinetic energy $E_{c i n}$ (down-left) and total energy $E_{t o t}$ (down-right)for the polytropic stars with different rotation rates. Red, black, blue and purple lines correspond to angular velocities $\Omega_{0}=0.026,0.052,0.104,0.260$ respectively.

In order to see if rotation could be of importance to break the spherical symmetry of this test, we repeated the calculation using different amounts of initial angular momentum (assuming rigid body rotation around the $Z$ axis). Figure $\square$ shows the internal, potential, kinetic and total energy of such tests which indicates that increasing the initial angular momentum implies bigger oscillations (amplitudes) and a shift on the time the first minimum occurs in the internal energy. Figure 3$]$ shows the total angular momentum and the column density along the $Y$ axis after 30 dinamical time scales. Overall, the results were qualitatively similar to the tests with no rotation, showing that the code could properly evolve such configurations without generating huge instabilities. All these tests were 

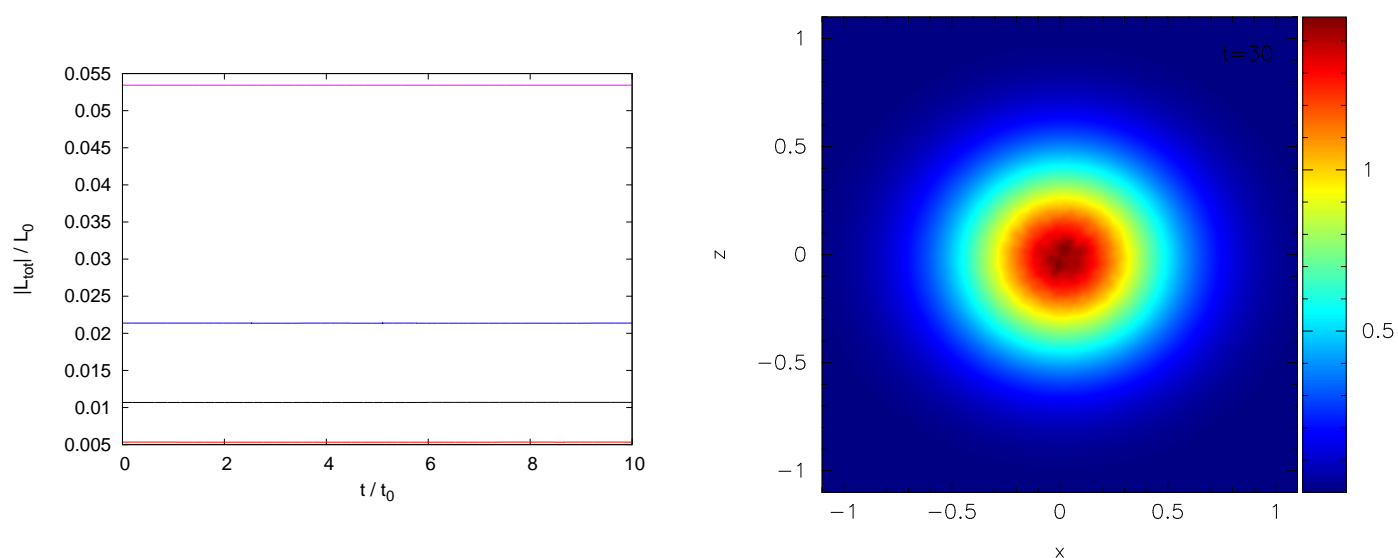

Figure 3: Total angular momentum for tests with different initial rotation rates (left) and column density along the $Y$ axis for the second slowest polytropic star $(\Omega=0.52166)$ after 30 dynamical time scales (right). Rotation creates a small deviation from spherical symmetry.

made using $5 \times 10^{4} \mathrm{SPH}$ particles given that previous results showed this resolution properly solved the shock after collapse and tests with 10 times this resolution showed no significant changes.
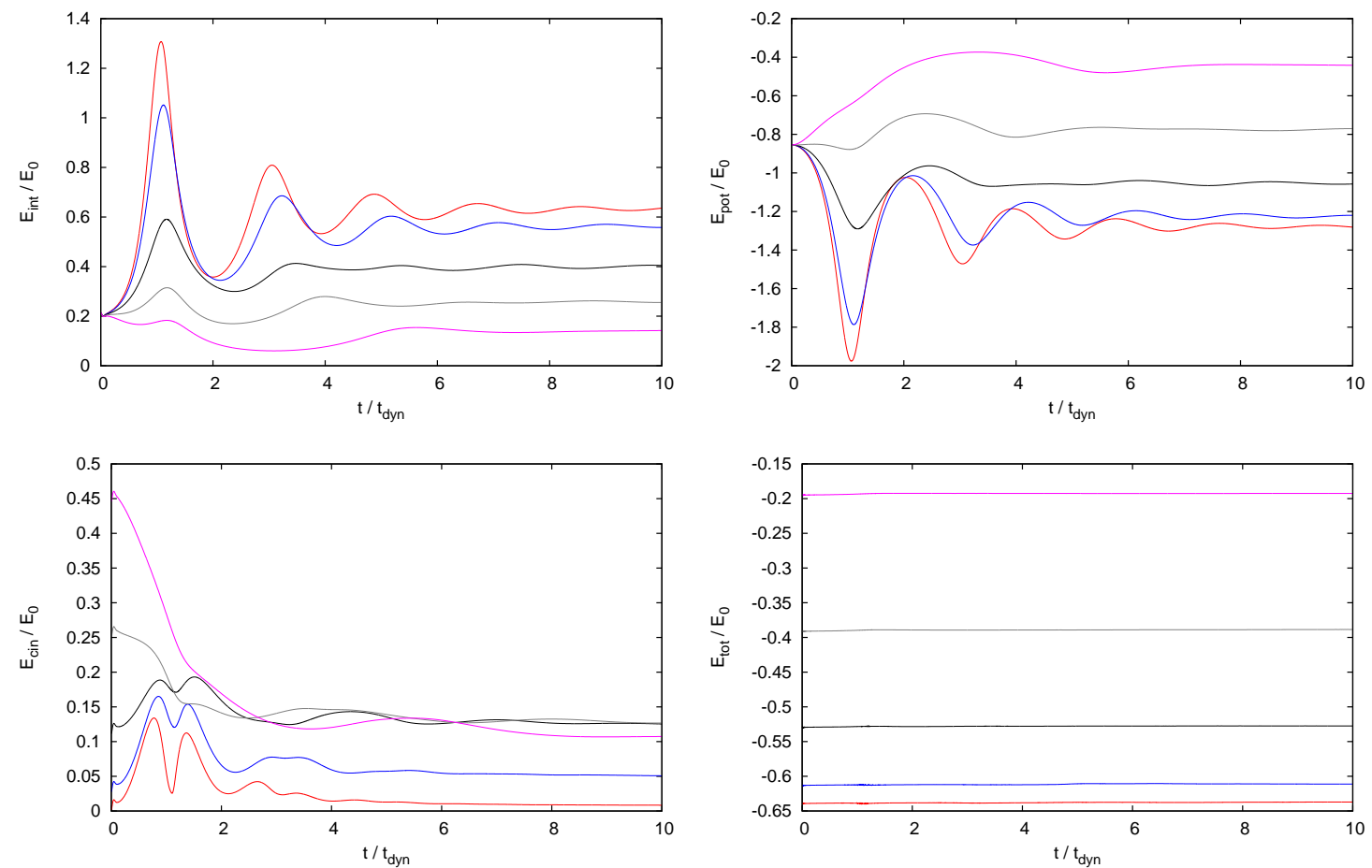

Figure 4: Potential (up-right), internal $E_{\text {int }}$ (up-left), kinetic (down-left) and total (down-right) energy plots for the collapse of $\gamma=5 / 3$ polytropes with low internal energy (half the value needed for a stable solution $E_{\text {int }}=E_{s t} / 2$ ). Each curve represents different rotation rates (rigid body angular velocity); purple line $\Omega=$ 2.0866, gray line $\Omega=1.5650$, black line $\Omega=1.0433$, blue line $\Omega=0.52166$ and red line $\Omega=0.13041$.

Working towards a collapsar model we proceeded to study the collapse of such polytropic stars 
with different rotation rates, by removing half the internal energy $E_{s t}$ needed for a stable solution (i.e. decreasing the pressure). In Figure $⿴$ we show collapses for different rotation rates (assuming a rigid body angular momentum distribution) and how increasing angular momentum translates into a shallower collapse shown on the potential and internal energies (figure 4 top panels).

\section{Collapse and Accretion in Slowly Rotating Polytropes}

In the context of the collapsar model, we tested the collapse and accretion of rotating polytropic stars considering that the innermost $1 M_{\odot}$ formed a $\mathrm{BH}$, with the outer layers collapsing due to the lack of pressure support. To account for accretion, we included a sink particle in the code, with an accretion radius $r_{a c c}=\eta G M_{B H} / c^{2}$, where $\eta>2$ determines how far from the Schwarzschild radius accretion takes place and the gas particles are swallowed by the $\mathrm{BH}$. Here we considered a value of $\eta=10$.
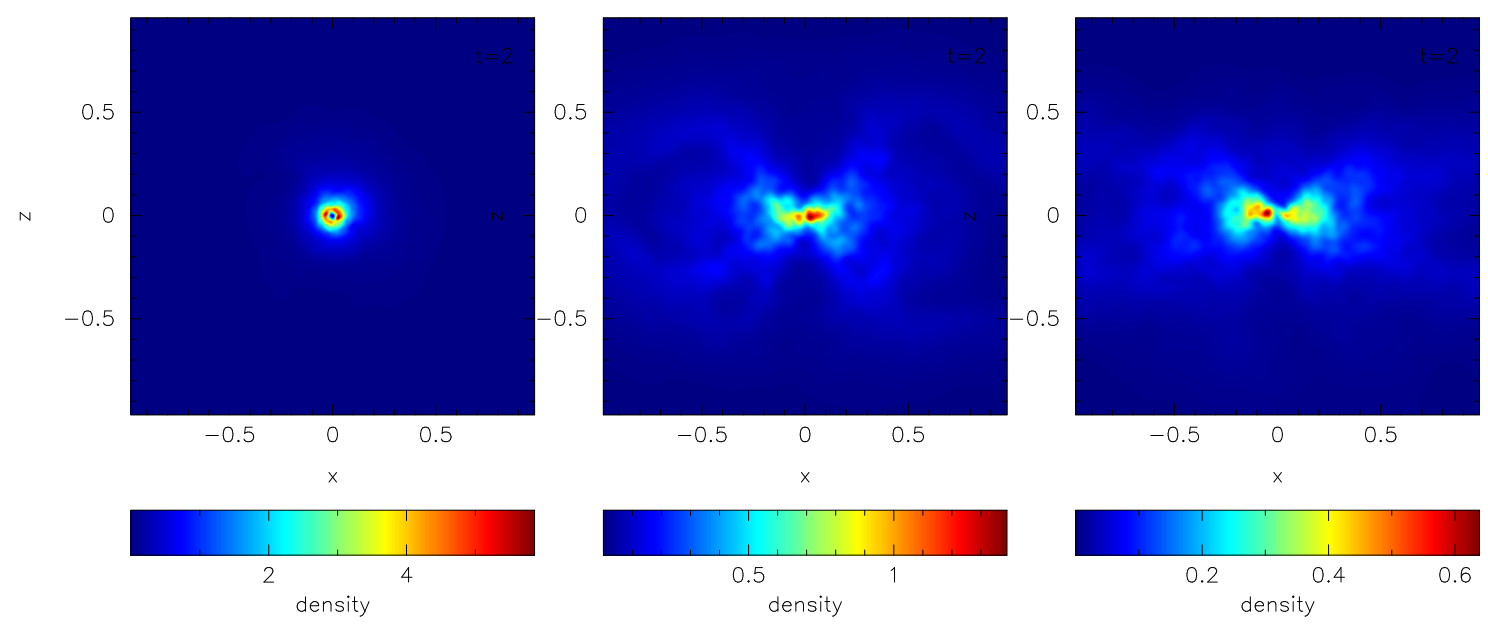

Figure 5: Density on the $X Z$ plane for polytropes with different rotation rates after $t=2 t_{d y n}$. The formation (and size) of the accretion disk depends on the initial angular momentum distribution given by $\Omega_{0}=0.104331,0.93898$ and 1.8258 from left to right.

\begin{tabular}{c|c|c|c|c}
\hline$\Omega_{0}$ [Nat. units] & $J_{0}$ in CGS & $r_{c}$ in CGS & $r_{c}$ [Nat. units] & $r_{a c c}$ [Nat. units] \\
\hline 0.1043 & $5.27 \times 10^{15}\left[\mathrm{~cm}^{2} \mathrm{~s}^{-1}\right]$ & $2.09 \times 10^{5}[\mathrm{~cm}]$ & 0.001 & 0.01 \\
0.9389 & $4.58 \times 10^{16}\left[\mathrm{~cm}^{2} \mathrm{~s}^{-1}\right]$ & $1.58 \times 10^{7}[\mathrm{~cm}]$ & 0.12 & 0.01 \\
1.8258 & $9.24 \times 10^{16}\left[\mathrm{~cm}^{2} \mathrm{~s}^{-1}\right]$ & $6.31 \times 10^{7}[\mathrm{~cm}]$ & 0.48 & 0.01 \\
\hline
\end{tabular}

Table 1: Initial specific angular momentum $J_{0}$ for the innermost material and its corresponding circularization radius $r_{c}$. The model with $r_{C}<r_{a c c}$ (and also $J<J_{\min }$ ) shows spherical accretion and no disk is appreciated.

As we can see from Figure [, if rotation is too slow the infalling material cannot form an accretion disk around the $\mathrm{BH}$, and we end up with Bondi like accretion. On the other hand, increasing rotation results in the formation of accretion disks of different sizes and densities. Considering that the gas will eventually get into a circular keplerian motion around the $\mathrm{BH}$ at a distance $r_{C}$, we 
can estimate this circularization radius $r_{C}$ in terms of the initial specific angular momentum of the innermost material:

$$
r_{C} \simeq J_{0}^{2} / G M_{B H}
$$

On table $\mathbb{W}$ we show an estimate of the circularization radius for polytropes with different rotation rates shown on Figure $\left[\right.$. Notice how that the slowest polytrope has $r_{C}<r_{a c c}$, this prevents the gas from forming a keplerian disk around the BH before being accreted. There is another condition for disk formation around a $\mathrm{BH}$ given by the innermost stable circular orbit at $R_{i s c o}=3 R_{g}$. To stay in this orbit the gas must have a minimum angular momentum $J_{\min }=6 G M_{B H} / c$. For a BH with $M_{B H}=1 M_{\odot}$ the minimum angular momentum is $J_{\min }=2.6575 \times 10^{16} \mathrm{~cm}^{2} \mathrm{~s}^{-1}$. So far we have only considered a Newtonian potential for the $\mathrm{BH}$ but to account for the existence of this innermost stable circular orbit we must consider a pseudo relativistic potential for the $\mathrm{BH}$.

\section{Modifications to the BH Potential}

In order to account for some of the relativistic effects on the gas, we included a PaczynskiWiita (PW) potential for the $\mathrm{BH}$. We tested the PW potential by observing the collapse and accretion of a $1 M_{\odot}$ non-rotating, polytropic envelope with very low internal energy (to avoid dispersion on the accelerations due to pressure gradients) and taking into account its self-gravity to compare its acceleration due to the $1 M_{\odot} \mathrm{BH}$ potential.

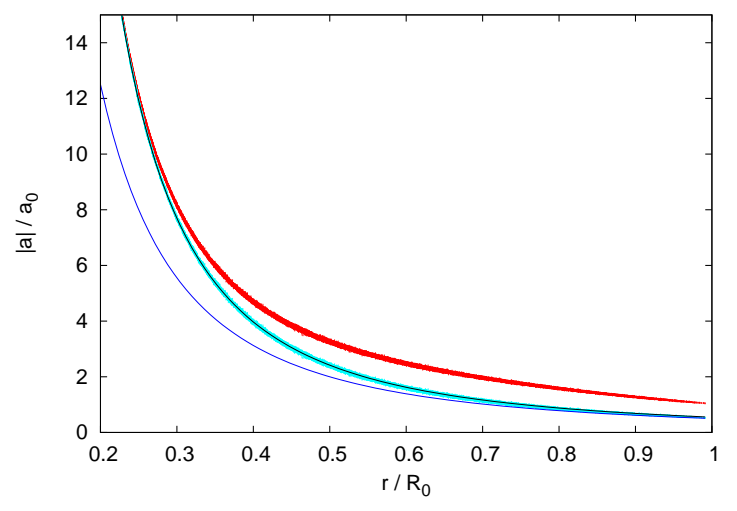

Figure 6: Acceleration of gas particles with low internal energy, falling into a $1 M_{\odot} \mathrm{BH}$ with a Paczynski-Wiita potential. The blue solid line and the black solid line represent the Newtonian and the PW accelerations (respectively) of a test particle, due to the BH. The red dots represent the acceleration of gas particles (considering self-gravity), and the cyan dots represent the acceleration of gas particles due only to the $\mathrm{BH}$ (without self-gravity).

As seen in Figure 6, the gas acceleration due to the $\mathrm{BH}$ (cyan dots) agrees with the acceleration expected due to a $1 M_{\odot} \mathrm{BH}$ with a PW potential (black solid line). Now that the PW potential has become fully functional, the gas would be able to reproduce the position of the innermost stable circular orbit at a distance $R_{i s c o}=6 G M_{B H} / c^{2}$ from the BH. So if we want to include this effect on the gas motion we have to consider an accretion radius $r_{a c c}$ equal or smaller than $R_{i s c o}$. From table [, and Figure [], we see that both the polytropes that formed a disk around the BH, have initial specific angular momentum $J_{0}$ bigger than the minimum angular momentum $J_{\min }$ needed to stay on the innermost stable circular orbit. On future calculations we must include information about the circularization radius $r_{C}$ observed on the simulations, the expected $r_{C}$ from the initial angulal momenutm $J_{0}$, and a comparison to the breakup angular momentum needed for the star to disrupt by its own rotation. This information will lead us to a more realistic collapsar model. 


\section{Summary}

These tests have shown that the code GADGET-2 [U] is able to solve properly the collapse and accretion of a polytropic envelope into a $\mathrm{BH}$, and have taken us closer in constructing the collapsar model. Results show that given enough angular momentum, the gas would create an accretion disk near the $\mathrm{BH}$ but, in order to be accreted, the material from the disk will have to lose angular momentum, and this depends directly on how efficient the angular momentum transport from the inner, to the outer parts of the disk (viscosity) is. At the time, we have not considered any other viscosity that the one coming from the SPH formulation numerically, but this would definitely be an issue to explore in future modifications. Further work will focus on including some simple cooling of the gas in order to observe its importance on determining the disk properties and the development of instabilities.

\section{Acknowledgments}

Support from CONACyT (83254 and a graduate fellowship for AB), the TEXAS Symposium 2010 LOC, and assistance from A. Rodriguez-Gonzalez with Gadget-2 is gratefully acknowledged, as is the use of SPLASH v.1.13 by D.J.Price.

\section{References}

[1] V. Springel, The cosmological simulation code GADGET-2. Mon. Not. R. Astron. Soc., 364, 1105-1134 (2005) arXiv:astro-ph/0505010v1

[2] V. Springel, N. Yoshida \& S.D.M. White, GADGET: a code for collisionless and gasdynamical cosmological simulations New Astronomy, 6, 51 (2001) arXiv:astro-ph/0003162v3

[3] A.I. MacFadyen \& S.E. Woosley, Collapsars: GRBs and explosions in "Failed Supernovae". APJ, 524 : 262-289 (1999) arXiv:astro-ph/9810274v1

[4] S.E. Woosley, GRBs from Stellar Mass Accretion Disks Around Black Holes. APJ, 405 : 273-277 (1993)

[5] M.A. Abramowicz, The Paczynski-Witta potential. A step-by-step "derivation”. A\&A 500, 213-214 (2009)

[6] D.Lopez-Camara, W.H. Lee \& E. Ramirez-Ruiz, GRBs and Supernova Signatures in Slowly Rotating Collapsars. Astrophys.J.692:804-815 (2009) [arXiv:astro-ph0808.0462v2]

[7] B. Paczynski \& P.J. Wiita, Thick accretion disks and supercritical luminosities. A\&A 88, 23-31 (1980)

[8] S.E. Woosley \& A. Heger, The Progenitor Stars of Gamma Ray Bursts. APJ, 637 : $914-921$ (2006) arXiv:astro-ph/0508175v1

[9] G. Rockefeller, C.L. Fryer \& H. Li Collapsars in Three Dimensions. arXiv:astro-ph/0608028v1 (2006)

[10] D.J. Price, SPLASH: An Interactive Visualisation Tool for Smoothed Particle Hydrodynamics Simulations. Publ. Astron. Soc. Aust. 24, 159-173. (2007) arXiv:astro-ph/0709.0832v1 